indivis, après avoir été dégagé de l'illusion unitaire comme monde, se donne essentiellement (p. 225) dans la pratique finie comme transcendance non-thétique, Autre non-positionnel (p. 224). Il y a un principe immanent d'agir, irréflexif indédoublable - . Le Monde, qui n'est pas une illusion, est le signal/support (p. 235) du sens (p. 243) ou de l'Autre non-thétique.

C'est un grand livre assurément qui justifie pleinement l'avertissement de l'auteur : « il y a là « matière à critique », mais on souhaite qu'elle ne dissimule pas la réalité de l'entreprise " (p. 6). La critique devra se justifier autant que l'ouvrage qu'elle visera, sous peine d'hallucination. Qui plus est, l'écriture est sobre, racée. L'ensemble respire d'ailleurs la puissance et la sérénité ; celles-ci sont rendues totalement effectives par la vigilance et l'exigence théorique interne les plus aiguës, les plus sceptrales, les plus " réelles ".

Serge VALDINOCI.

HISTOIRE DES SCIENCES ET ÉPISTÉMOLOGIE

Brian EASLEA, Science et philosophie. Une révolution, 1450-1750. La chasse aux sorcières. Descartes. Copernic. Kepler. Trad. de l'anglais par Nina GODNEFF. Paris, Ramsay, 1986. $15 \times 25,336$ p., index.

Cet ouvrage d'un historien britannique des sciences a paru en 1980 sous le titre plus exact de Witch-Hunting, Magic and the New Philosophy (Chasse aux sorcières, magie et philosophie nouvelle). Je remarque que le sous-titre de la traduction française qui précise quelque peu le titre, a le tort d'y ajouter l'incroyable défilé suivant qui est certainement une invention délirante de gallocentrisme : « Descartes. Copernic. Kepler ». Oublions ce bouleversement pseudo-chronologique où l'auteur n'est certainement pour rien et soulignons l'intérêt d'un travail qui, transplanté dans le milieu francophone de l'histoire des sciences, y détonne heureusement. On n'a guère l'habitude, en effet, dans ce milieu, de s'interroger sur la différence et l'inégalité traditionnelle des sexes, sur la misogynie magique de la chasse aux sorcières, sur la misogynie égale, mais moins trouble, de la science moderne, sur les rapports des croyances démonologiques d'une part, des doctrines mécanistes d'autre part, avec les inégalités sociales et l'opposition des pauvres et des privilégiés dans des contextes successifs de peur traumatisante de la nature et de confiance en sa réduction à un mécanisme conquérant. $\mathbf{M}$. Easlea se garde notamment de prendre pour argent comptant l'identification classique du mécanisme avec une sorte d'épanouissement de l'intelligibilité en matière de sciences de la nature, thèse qui veut ignorer des conceptions mécanistes l'audace souvent extravagante, les refus parfois insoutenables, les échecs et les obscu- 
rités, pour ne retenir que leurs triomphes, et affirmer, avec l'aplomb d'un manque total d'esprit historique, l'avènement de la Raison et l'accession à la Vérité ou la sortie définitive hors des ténèbres de l'ignorance et de l'absurdité.

Le besoin de comprendre selon quelles transformations s'est produite, dans la société européenne, l'exaspération de la chasse aux sorcières, suivie de son déclin et de sa disparition (l'exaspération : 1580-1650, disparition quasi complète : vers 1670 en France) n'empêche pas l'auteur de décrire les traits conceptuels de la cosmologie aristotélicienne, puis de l'astronomie de Ptolémée et de la révolution marquée par le nom de Copernic, puis par ceux de Galilée, de Kepler, de Descartes, de Newton (de ces quatre, le premier et le dernier exclus de la couverture, on ne sait trop pourquoi). Ces chapitres paraîtront moins originaux au lecteur français, encore que ce qui est dit de Descartes a propos de son explication mécaniste du magnétisme manifeste un souci de précision peu fréquent de ce côté-ci de la Manche, où les Principia cartésiens ne sont que peu étudiés. La réduction de certaines « sympathies " à des explications mécanistes du style de la magie naturelle est bien vue (les plaies de l'assassiné qui se rouvrent à l'approche de l'assassin, cf. p. 138, voir aussi p. $35,146,166$, sont « réinterprétées » dans la traduction française des Principes, IV, $\mathrm{n}^{\circ} 187$, cf. ma note à ce sujet, Revue philos., 1984, p. 219-220). C'est que l'auteur se montre attentif aux accommodements non moins qu'aux conflits de la mentalité démonologique avec la magie naturelle dont le représentant le plus éminent, le chancelier Bacon, est peut-être trop sommairement évoqué, bien que son accord avec la religion et sa conception utilitaire de la science nouvelle soient soulignés à bon droit.

" Il n'était nullement évident, écrit B. Easlea, dans les années 1650, que les tenants de la magie naturelle purifiée, ou philosophie mécaniste, finiraient par l'emporter - ce qui conduit à s'interroger sur les raisons de la victoire, au tournant du siècle, de la cosmologie fort peu plausible des philosophies mécanistes et du succés de la théorie préformationniste de l'emboftement " (p. 11). La réponse (ibid.) tient essentiellement en ceci : « la disparition des procès de sorcellerie au cours de la seconde moitié du XVII* siècle s'explique par la stabilité et l'assurance croissante des classes privilégiées [...] et par la volonté bien arrêtée de s'approprier le monde physique. " Le mécanisme, envisagé d'un point de vue purement théorique, gagne en effet contre l'horreur du vide, mais s'épuise en subtilités contre le magnétisme, dès les débuts de sa lutte. Finalement, il doit céder la place à l'attraction gravitationnelle dont la science newtonienne reconnaît d'expérience la réalité et la détermine avec rigueur, en conservant du mécanisme seulement l'esprit nouveau de conquête et de maîtrise, tout en en dépassant les limites, c'est-à-dire ses préjugés théoriques et son recours insuffisant aux opérations de mesure, par l'emploi systématique d'un mathématisme expérimental issu, moins paradoxalement qu'il n'y paraît, de la persistance d'une tradition baconienne, il est vrai élargie par l'enseignement de l'école de Galilée et aussi de celle de Descartes, non moins que de Kepler.

Ce qu'il convient de mettre en relief dans la réflexion généralement bien documentée de l'Auteur, c'est ce fait remarquablement peu perçu dans l'école française d'histoire des sciences que le triomphe, puís le dépassement des conceptions mécanistes non seulement se sont accompagnés de luttes sociales 
d'une extrême dureté, mais même en ont été largement conditionnés. Le mécanisme, nous dit l'Auteur, n'apportait pas seulement certaines valeurs de clarté et de puissance d'explication, ainsi qu'il le reconnaît volontiers ; cette philosophie " était généralement regardée comme une philosophie "antisubversive", soutenant la religion et l'ordre social face à la menace de la magie naturelle et des "illuminés" [...] elle légitimait et faisait apparaître comme possible l'appropriation du monde naturel sans mettre en cause la nature miraculeuse des cuvres du Christ " (p. 239). Au contraire, la « magie naturelle " non purifice, comme dit B. Easlea, c'est-à-dire soit naturaliste, soit plus ou moins illuminee, avait partie liée, notamment pendant la Révolution anglaise, avec les mouvements de révolte des déshérités, Levellers (Niveleurs) et autres, parfois athées, souvent égalitaristes, toujours dangereux aux yeux des possédants ; quelle que fût leur éventuelle religiosité, les uns niant l'existence du diable, les autres gardant des liens plus étroits avec les croyances démonologiques (communes aux sorcières et à leurs persécuteurs, c'est-à-dire à des déshérités de sexe principalement féminin et aux nantis d'avant les solutions mécanistes), ils représentaient une menace de subversion sociale pour les classes dirigeantes. Il est significatif qu'à peine débarrassé, après des hésitations, de Charles Ier, Cromwell, en 1649, écrase les Niveleurs. Les grandes doctrines en apparence les plus éthérées n'organisent pas seulement de purs concepts et l'on risque de ne pas bien comprendre ces concepts eux-mêmes si l'on fait abstraction des forces sociales qui les soutiennent et contribuent à leur conférer une trouble et precaire évidence en masquant leurs limites et leurs difficultés théoriques.

Quelques erreurs ou insuffisances appellent pourtant des rectifications. Ainsi, la glande pinéale, centre du cerveau pour Descartes, est ce qu'on nomme aujourd'hui l'épiphyse et non l'hypophyse (p. 145). Plus importante est la présentation erronée de l'expérience du Puy-de-Dôme : il n'est pas sûr que l'idée en soit venue à Pascal plutôt qu'a Descartes, ce qui est secondaire, la création essentielle restant en la matière la propriété de Torricelli. Ce qui est súr en revanche et capital est ceci : $1^{\circ}$ ) Descartes a nettement prévu le résultat ; $2^{\circ}$ ) ce résultat ne l'a nullement dérangé dans ses convictions de partisan du plein. Bel exemple contre tout " experimentum crucis ", dont on ne trouve plus aucune trace dans l'exposé de l'expérience tel que l'offre l'ouvrage '. D'autre part, s'il n'avait tenté de dépeindre Thomas Hobbes sous les traits d'un athé, dans la ligne des ragots d'époque et à l'encontre de tout ce que l'on peut tirer des textes mêmes de l'intéressé et de ses relations amicales avec Mersenne et Gassendi, sous les traits aussi d'un matérialiste (cf. p. 196-197), alors que son mécanisme a absolument besoin d'un ordonnateur suprême et que son pseudo-matérialisme est unifié sous une nécessité venue d'en-haut, l'auteur aurait mieux situé, en plein accord avec sa thèse d'ensemble, la pensée de Hobbes du côté des privilégiés auxquels le rattachaient ses liens avec la cour de Charles Ier, du côté des mécanistes contre les « méchaniques », les déshérités, les « manuels ». Il est vrai que la position

1. Les pièces du dossier et les savants commentaires de M. Jean Mesnard sont dans sa magnifique édition des CEuvres complètes de PAscal, t. II, Paris, Desclée de Brouwer, 1970. 
originale d'un philosophe d'esprit à la fois religieux et rationaliste, hostile au droit divin des rois, individualiste pour mieux aboutir à un autoritarisme absolu, étranger en fin de compte à toutes les orthodoxies, avait beaucoup de chances de dérouter ; l'illustre historien Christopher Hill, que B. Easlea prend pour inspirateur (p. 12), a précisément confondu pendant un certain temps « mécanistes " et "méchaniques ", mais il a par la suite reconnu l'erreur qui consistait à assimiler la philosophie mécaniste avec le radicalisme des éléments subversifs 2 . Le cas de Hobbes illustre fort bien la légitimité de cette correction, que l'auteur ne semble pas avoir connue. En tout cas, d'apres la traduction, le rationalisme de Hobbes est mis en question à partir d'un contresens flagrant (p. 193), où il est affirmé au nom de Hobbes que les mystères de la religion chrétienne vont « contre la Raison " (guillemets dans le texte ; Hobbes dit le contraire a deux reprises, trad. Tricaud, p. 117 et 396, texte de MacPherson, p. 180 et 410 , Leviathan, chap. 12 et 32 ).

On discerne dans la traduction quelques imperfections (notamment p. 105, ligne 5 à partir du bas, un imparfait du subjonctif bizarre), mais l'ensemble paraît fidèle aux intentions de l'auteur qui se proposait (p. 10) de fournir « un ouvrage de référence utile pour les cours d'initiation à la philosophie des sciences ». Équilibré et complété par d'autres, le livie de B. Easlea fera réfléchir et remplira son office ${ }^{3}$.

Jean BERNHARDT.

Marco Messeri, Causa e spiegazione. La Fisica di Pierre Gassendi. Milano, Franco Angeli, 1985. $14 \times 22,170$ p. ("Collana di filosofia $», 230-18$ ).

Dans cette étude consacrée à la physique de Gassendi, Marco Messeri se propose d'illustrer quelques aspects de l'image de la science qui y est contenue, et de déterminer les liens qu'elle entretient avec la pratique scientifique. L'apport de Gassendi à la science de son temps a été diversement évalué, en fonction méme de la conception que l'on avait de la révolution scientifique du XVIı siecle. L'évaluer justement implique que l'on ait présente a l'esprit la complexité du panorama des sciences, la pluralité des modèles, la complexité des méthodes cognitives et leur diversité, tout comme celle des méthodes effectives mises en cuvre. Très attentif à eviter toute perspective réductrice, l'Auteur rappelle qu'il est du rôle de l'historien de tester la valeur et la cohérence des arguments et des preuves et préfère parler des « images de la science " pour souligner la variabilité historique des critères scientifiques.

2. Cf. Christopher Hitl, Le Monde d̀ l'envers, Paris, Payot, 1977, p. 230 (The World Turned Upside Down, London, 1972).

3. On doit regretter l'absence de table des matières. 
La méthode de Gassendi entretient avec sa conception de la nature un lien particulièrement fort et implique l'analyse de ses idées sur la structure physique du monde.

Entre le De Veritate, ouvrage de polémique contre Cherbury, la Disquisitio Metaphysica qui répond à Descartes, les Exercitationes, et le Syntagma Philosophicum, M. Messeri discerne, non un simple changement de ton, mais une réelle évolution. Sous l'influence des études qu'il consacre de 1640 à 1642 à la mécanique de Galilée, Gassendi est conduit à dépasser le scepticisme qu'il opposait à la physique de l'École, et se trouve confronté à des problèmes tels que l'empirisme des Exercitationes ne suffit plus; la science ne peut plus se borner à la description, mais doit passer à l'explication des phénomènes, reconduire à « la nature interne » des choses, à l'essence, source des propriétés et des opérations ". La vérité de l'expérience fondait les exigences limitées de la " scientia experimentalis ", la vérité des sens - qu'il vient d'établir contre l'attitude qu'il juge maintenant excessive sur ce point des sceptiques - vient fonder l'édifice des sciences naturelles. La nouvelle mécanique exigeait des critères plus rigoureux que ceux de l'empirisme, Gassendi les trouve dans « l'observation intersubjectivement contrôlée ". Dans la Disquisitio Metaphysica, il opposait à Descartes le fondement sensible de toutes les idées - scientifiques ou vulgaires — ; il admet dans le Syntagma Philosophicum la connaissance discursive d'objets ultrasensibles comme Dieu et l'âme immatérielle.

Cette évolution de la théorie, pour Marco Messeri, véritable transformation des positions épistémologiques de Gassendi, lui permet de dépasser le fidéisme des Exercitationes et de légitimer l'enquête sur les causes des phénomènes physiques. Se met alors en place un savoir limité mais réel, une science conjecturale dont la voie est celle de la solution problématique, posée d'emblée comme connaissance imparfaite opposée à un savoir vrai - qui n'est pas décrit - mais inaccessible à l'homme, comme l'est pour Gassendi l'évidence cartésienne qu'il estime dépourvue d'utilité pour la connaissance des phénomènes physiques.

Contrairement aux traditions interprétatives qui voudraient l'en tenir éloignée, l'Auteur souligne la solidarité des enjeux de la physique de Gassendi avec ceux de la révolution mécaniste, quelles que soient par ailleurs les différences de contenu et de méthode : le faible pouvoir unifiant, la défiance à l'égard de la mathématisation. Cette physique rompt avec l'univers d'Aristote et lui substitue un espace infini et homogène, où l'espace et le temps sont perçus comme « cadres vides ", " théâtre " des phénomènes; les concessions à la physique de l'École sont purement verbales. Par ailleurs, la nature féconde et dynamique de Gassendi n'est pas celle des philosophes de la Renaissance ; l'astrologie comme la conception d'une nature prodigieuse lui paraissent résolument étrangères à la démarche de la science moderne.

L'effort, pour montrer comment s'intègre à la révolution scientifique la contribution de Gassendi, amène l'Auteur à en réexaminer la cohérence qu'il soutient contre les interprétations qui y voient une physique faite de « pièces et de morceaux " ou le produit d'un éclectisme impuissant à la synthèse ; il s'attache à montrer comment mécanisme et dynamisme, mécanisme et vitalisme s'intègrent sans s'opposer à sa conception de la « machine du monde ». 
La formulation correcte du principe d'inertie publiée pour la première fois par Gassendi, jugée incohérente avec le reste de sa physique, ne remet pas en question, aux yeux de l'Auteur, la vaste construction du Syntagma Philosophicum et l'incohérence demeure limitée. Causalité mécanique - loi d'inertie - et causalité interne - force motrice des atomes que Dieu leur a accordée - se combinent dans la notion de mécanisme comme essence. La nature interne rend compte de l'origine du mouvement, la nature externe, de sa transformation sous l'effet de la loi des chocs. Le mouvement n'est pas externe et communiqué comme dans le mécanisme cartésien mais interne et naturel, l'effet d'un conatus. La clé de voûte de la mécanique de Gassendi réside dans l'idée que tout mouvement sensible prend sa source dans le mouvement des atomes. En droit, l'être étant tout entier de l'ordre du visible, le mécanisme interne serait accessible à une vision rendue plus parfaite par l'appareillage scientifique, et Gassendi reconnaît à la physique la mission de connaître le mécanisme interne qui rend compte des effets observables.

Dotant le « pondus " des atomes d'une « vis sese movendi », il produit, sans se mettre en contradiction avec le mécanisme des anciens, une theorie du mouvement cohérente et clairement définie.

Pas plus qu'entre mécanisme et dynamisme, l'Auteur ne voit entre mécanisme et vitalisme une alternative irréductible. Les « semina rerum » (identifiés avec les molécules, ou agrégat d'atomes) rendent compte du développement des êtres vivants comme des structures internes de la pierre et du cristal dans une physique où prédomine le modèle du vivant, où est gommée toute coupure nette entre l'organique et l'inorganique. La conception d'une matière doté de force, finalisme et mécanisme se combinent sans contradiction dans la représentation d'une nature active et féconde où l'acte créateur de Dieu a introduit la force, l'efficace et l'énergie. Une nature crée de telle sorte qu'elle ne nous soit pas, du tout, inintelligible.

Ni l'attachement à l'érudition, ni le fait que la méthode et la structure conceptuelle de sa physique (peu unifiante, non idéalisante et mathématisante, comme on l'a noté) soient plus proches des « sciences baconiennes " que de la conception mécaniste de la nature, de Galilce, de Descartes ou de Newton, ne peuvent suffire à l'écarter du combat multiforme, mené par la science moderne à son avènement. Au contraire, comme bien des contemporains, l'Auteur voit en lui un allié de Descartes et de Mersenne sur les deux fronts où sont engagés le mécanisme, la physique de l'École, et la nature foisonnante des penseurs de la Renaissance.

L'ouvrage de Marco Messeri, en même temps qu'une étude éclairante de la physique de Gassendi, apparait comme une contribution importante au mouvement, déjà largement amorcé, qui tend à rendre à la révolution scientifique du XVII' siècle toute sa richesse et sa diversité, restituant à côté du mécanisme cartésien bien d'autres images de la science.

Françoise Charles-DauberT. 
Enseignement et diffusion des sciences en France au XVIIr siècle. Sous la dir. de René TAton. Paris, Hermann, 1986. $14 \times 21,778$ p., annexes, bibliogr., index (" Histoire de la pensée ").

Plus de trente ans ont passé depuis que René Taton et ses collaborateurs ont entrepris le projet qui a été réalisé dans la première édition de cet ouvrage, publié en 1964. À cette époque, l'histoire sociologique et même institutionnelle des sciences était dans son enfance. Aucun autre ouvrage n'a plus contribué à son passage à la maturité en encourageant et facilitant des recherches ultérieures. Mais l'effet fut produit plutôt en donnant l'exemple qu'en portant un défi. L'époque et le milieu, la France au siècle des Lumières, étaient particulièrement propices. Il restait une unité de culture assez cohérente pour que l'entré des matières d'ordre technique dans la République des Lettres puisse fournir le thème. Néanmoins, la variété et la vitalité des institutions créées par l'ancienne Monarchie ne sauraient qu'étonner le lecteur de nos jours. Un des grands mérites de l'ouvrage réside dans le fait que les auteurs ont su, au-delà des formes administratives et institutionnelles, découvrir autant que possible le contenu de ce qui était enseigné dans la plupart de ces écoles. Il existe certainement très peu d'œuvres pionnières si solides qu'elles restent utiles, et même indispensables, dans le domaine d'études qu'elles ont en grande partie lancé. Mais tel est le présent cas. Mon propre exemplaire de la première édition est presque usé ainsi que celui qui se trouve dans la bibliotheque universitaire. Cette seconde impression sera d'autant plus appréciée que l'éditeur a eu l'idée heureuse d'imprimer, en même temps et en fascicules séparés, les cinq parties principales suivantes, qui peuvent servir de manuels d'enseignement :

- Ch. Bedel, R. Hahn, Y. Laissus, J. Torlais, La Curiosité scientifique au XVIII siecle : cabinets et observatoires;

- Ch. Bedel, P. Huard, Médecine et pharmacie au XvIIr siècle ;

- A. Birembaut, F. de Dainville, P. Gille, F. Russo, G. Serbos, Ecoles et techniques militaires au XVII/ siecle;

- P. Costabel, F. de Dainville, M. Lacoarret, R. Lemoine, $\mathbf{M}^{\mathrm{me}}$ Ter Menassian, L'Enseignement classique : collèges et universités au XVIII' siècle;

- Y. Laissus, J. Torlais, Le Jardin du roi et le Collège royal.

Charles C. GiLlisPie.

G. Th. Guiltbaud, Leçons d'à peu près. Paris, Christian Bourgois, 1985. $13,2 \times 20,235$ p., index.

Ce livre rassemble des textes qui furent objet d'articles ou d'enseignement oral et transmet au moins une part de l'expérience d'une vie. L'auteur a cédé à l'insistance de ses amis, collègues et auditeurs, pour rendre accessible à un public étendu le faisceau des illustrations d'un thème de réflexion particulièrement important. 
Les illustrations étaient dispersées, dans le temps et dans l'espace bibliographique ; le thème, présent en toutes, avait besoin d'être dégagé avec la netteté requise pour l'information large. En revoyant ses « Leçons d'à peu près » pour une publication, l'auteur a heureusement répondu à l'ensemble des souhaits de tous ceux qui eurent profit à l'entendre. À l'entendre et à saisir combien la conception courante de l'exactitude des mathématiques souffre de la méconnaissance des réalités. À savoir que « la mathématique, loin d'exclure l'approximation, se l'approprie : elle en parle beaucoup, mais avec rigueur " .

Et il est bien vrai que " parler de l'à peu près avec rigueur » relève d'un " apparent paradoxe ", mais que c'est là une " tâche fondamentale " qui est depuis des siècles le moteur du progrès mathématique à travers les problèmes posés à l'esprit. Problèmes anciens de mesures, comparaisons, justes répartitions, qui ne font que se renouveler en leur forme au cours des siècles, mais aussi problèmes nouveaux et ardus que les sociétés modernes rencontrent dans leur recherche pour maîtriser le probable et le prévisible. Pour en parler avec efficacité, donc avec rigueur, il y faut une culture, considérable, et une compétence à la fois technique et philosophique. L'auteur possède l'une et l'autre, et son style, alerte, maintient avec le lecteur un dialogue coloré et plein de vie.

Car s'il a été durant toute sa carrière un surdoué de l'enseignement oral, il n'a pas cessé de parler en écrivant, et on l'écoute en le lisant, avec plaisir. Ce n'est pas, aujourd'hui, un mince mérite, et son livre ne souffre pas d'être fait de morceaux qui n'ont pas été recomposés au sens strict, mais seulement revus et corrigés. La mosaïque dans laquelle ils s'insèrent constitue effectivement un tableau et à les suivre, à constater comment ils s'articulent les uns aux autres, le lecteur n'a pas besoin qu'on l'accable de discours ; c'est dans une synthèse en acte qu'il est entraîné et qu'il entre de lui-même, poussé par l'éveil de l'intérêt.

À ce point de vue, les intentions de l'auteur sont satisfaites. Dans le bref « Avis au lecteur », il demande à celui-ci « patience et obstination » afin d'aboutir à « une idée personnelle » de la question, devant quelques données d'une enquête sans conclusion. C'est là une haute conception de l'écriture au service de la communication, et il convenait bien que le présent compte rendu souligne d'abord cette qualité rare, à travers laquelle la captatio benevolentiae n'est pas déçue.

Bien entendu il y a mieux à dire, mais dire cela, d'emblée, était aussi nécessaire pour qu'il n'y ait pas méprise. L'ouvrage n'a pas été écrit pour constituer un exposé savant ou érudit, ce n'est ni un traité d'histoire des mathématiques, ni un livre de technique mathématique ; il a été conçu et réalisé pour éveiller et susciter la réflexion. Mais il repose sur une masse impressionnante de références historiques, soigneusement présentées avec les notations critiques qui s'imposent, et, en définitive, il est une source étonnante d'informations contrôlées.

Tout ceci étant dit, il faut accorder quelques détails au contenu. On ne saurait s'étonner de ce que la mesure de la longueur de la circonférence ait fourni à l'auteur matière à illustrations. Le florilège de la question est considérable depuis la plus haute Antiquité, mais en dégager les éléments caractéristiques n'était pas superflu.

Il est certainement peu connu aujourd'hui du grand public que la Bible (Livre des Rois et Chroniques) parle du tour d'un bassin comme étant le triple du diamètre, et $\mathrm{G}$. Guilbaud a raison de signaler que cette approximation est encore 
aujourd'hui utilisée à l'occasion dans des débats très sérieux. Par exemple celui qui concernait en 1981 la construction d'un cyclotron près de Genève ; dix kilomètres de diamètre, trente kilomètres de tour. Quand il s'agit d'argumenter sur un encombrement, une approche grossière suffit. Et il est bien évident que l'approche qui consiste à prendre 3 pour le rapport $\pi$ est plus ou moins grossière suivant le problème technique à résoudre. L'auteur est bien inspiré de distinguer entre technique et mathématique. C'est la mathématique qui se préoccupe de préciser dans quel intervalle se situe le rapport de la circonférence au diamètre. Et il est bon, à cet égard, de rappeler le texte d'Archimède qui fixe l'encadrement entre $\frac{22}{7}$ et $\frac{223}{71}$. Les techniciens jugent de l'à peu près selon leurs besoins, le mathématicien met de l'ordre dans le jugement, c'est une excellente illustration.

Encore faut-il être scrupuleux. C'est avec raison que G. Guilbaud s'applique à ne pas trahir Archimède : "Pour tout cercle, le périmètre dépasse le triple du diamètre de moins d'un septième, mais de plus de dix septante et unième. " Lorsque nous traduisons que le rapport $\pi$ est donc chez Archimède plus petit que $3+\frac{1}{7}$ et plus grand que $3+\frac{10}{71}$, nous transformons à coup sûr. Non seulement en introduisant la notion du rapport $\pi$ qui a mis tant de temps à s'imposer, mais surtout en gommant des procédures de calcul dans lesquelles seules les fractions de l'unité étaient utilisées. La rigueur mathématique d'Archimède qui consiste à préciser l'encadrement d'une mesure a donc précédé de loin une traduction qui constitue pour nous un progrès, mais cette traduction oblitère quelque chose qui mérite encore aujourd'hui d'être connu.

Le fait est plus frappant peut-être avec la quadrature du cercle. G. Guilbaud rappelle que le papyrus $\mathrm{Rhind}$ (1700 avant J.-C.) contient l'évaluation de l'aire du cercle comme étant celle du carré construit sur le diamètre diminué d'un neuvième, et il souligne que la règle de calcul énoncée relève d'une finesse. Diminuer le diamètre d'un neuvième, en faire ensuite le produit par le diamètre, puis diminuer encore ce produit de son neuvième.

Il semble, toutefois, que l'auteur passe ici quelque peu à côté de la leçon qu'il a lui-même retirée du texte. Car le rédacteur du papyrus donne deux exemples d'application de la règle, à savoir d'abord un diamètre de 9 , puis un diamètre de 10. Le premier cas, qui donne $(9-1) .9-\frac{(9-1) .9}{9}=(9-1) \cdot(9-1)=64$, illustre que le résultat est bien le carré du diamètre diminué d'un neuvième. Mais le second est plus complexe. Pour le diamètre 10 , le papyrus donne seulement le résultat $79+\frac{1}{108}+\frac{1}{324}$ et $\mathrm{G}$. Guilbaud essaie pertinemment de le justifier en suivant les étapes de l'application de la règle. Il omet seulement de dire que ces étapes sont commandées par l'utilisation du neuvième, et de ses fractions comme de ses multiples. Ce n'était pas sacrifier à une lourde insistance que de déclarer ici avec toute la clarté requise comment le texte très ancien doit être lu. 
Il n'est pas sûr, en effet, que le lecteur perçoive instantanément que $79+\frac{1}{108}+\frac{1}{324}=79+\frac{1}{9.12}+\frac{1}{3.9 .12}$, ni que la procédure par neuvièmes et fractions de neuvièmes permet « d'arriver » aisément à ce résultat.

Quant à la question de savoir pourquoi le scribe égyptien n'a pas simplifié davantage et écrit $79+\frac{1}{81}$, c'est évidemment une très bonne question dont la réponse réside probablement dans les difficultés de manipulation de fractions, mais G. Guilbaud eût été bien inspiré de remarquer que de toute manière le scribe savait que 79 est une excellente approximation de l'aire du cercle de diamètre 10 .

S'il faut ainsi entrer dans un détail assez inhabituel pour le genre littéraire des comptes rendus, c'est que les regrets qu'il y a lieu de formuler viennent de la réflexion même que l'auteur sollicite chez son lecteur, et ces regrets sont liés à des mathématiques incompréhensibles sans un minimum de développement.

Et il faut, sur l'exemple qui vient d'être relevé, aller jusqu'au bout d'une critique amicale. Dans les pages qui font suite à cet exemple, G. Guilbaud poursuit l'information du public en tirant de travaux oubliés le témoignage d'écrits médiévaux, notamment celui de Francon de Liège. C'est bien intéressant. Au lieu de diminuer d'un neuvième, comme l'Égyptien, le calculateur du XI' siècle prenait un huitième. Était-ce mieux ou moins bien ?

La question méritait d'être posée. Mais si pour la résoudre il fallait bien accepter d'introduire $\pi$ pour écrire que la bonne fraction cherchée, $f$, répond à $(1-f)^{2}$ $=\frac{\pi}{4}$, on voit mal pourquoi on n'utilise pas les calculs déjà faits. À très peu près, l'Égyptien donnait $\left(1-\frac{1}{9}\right)^{2}=0,790$ et il est aisé de voir que $\left(1-\frac{1}{8}\right)^{2}=0,765$. Ce que nous savons de $\pi$ suffit alors à donner la préférence, largement, à $\frac{1}{9}$. À quoi bon chercher l'encadrement de $\frac{1}{\mathrm{f}}$ au moyen du calcul de $\sqrt{\frac{\pi}{4}}$ !

Et pourquoi aussi ne pas dire en clair que cet autre texte médiéval, qui se traduit par $\left(1+\frac{1}{4}\right)^{2}=\frac{\pi}{2}$, conduit à la valeur par défaut plus approchée de 3,125, meilleure que celle de Francon.

Finalement, et à propos de $\pi$, on ne saurait douter que la conscience de l'à peu près ait été éveillée durant très longtemps, en laissant des traces de procédés dignes de mémoire. Et il est très important que nous soyons remis en face de faits significatifs. Simplement, l'appel, que G. Guilbaud fait à la réflexion active de son lecteur, amène celui-ci à l'interpeller.

Que la redécouverte d'Archimède par l'Occident au XVI siècle ait été décisive pour la recherche mathématique des encadrements de $\pi$, que soit ainsi privilégié le calcul de périmètres de polygones réguliers et donc le calcul effectif de racines carrées, et tout un développement de la liaison entre pensée et technique mathématiques est bien situé. Bien situé et remarquablement analysé. Pourtant, d'où vient que l'approximation par quadrature, dont on a relevé de 
si bons témoins, n'ait pas été poursuivie ? Est-ce parce que toute tentative d'échapper par cette voie à la racine carrée se révèle rapidement vaine ? On aurait aimé que le grand chapitre, passionnant, que G. Guilbaud consacre à « Quarante siècles de racines carrées " soit orienté vers cette question.

«On n'évite pas les racines carrées » lit-on p. 76 ; ce trait fugitif manque de commentaires. L'auteur les fera peut-être un jour. Il a trop bien entraîné son lecteur à constater des faits, tous aussi curieux les uns que les autres, pour laisser ce lecteur sur sa faim. Que la mathématique moderne retrouve le privilège de la puissance 2 sous des formes nouvelles a quelque chose d'impressionnant. C'est une lueur sur une vérité fondamentale, mais cachée.

Cette remarque vaut pour la masse des autres considérations que ce petit ouvrage resserre dans une grande densité. Les "curiosités arithmétiques " dont le présent compte rendu vient d'évoquer une partie, ne sont elles-mêmes qu'un aspect de ces mathématiques sociales ou appliquées que G. Guilbaud a longuement méditées et où il puise des exemples nombreux et savants. On ne pouvait dire ici que ce qui est le plus immédiat est le plus facile. Rendre compte de tout serait écrire un autre livre, il faut se résoudre à suggérer seulement, et sur les points où il est possible d'être à la fois bref et clair, en quoi le livre - celui qui est écrit et livré au lecteur - est en fait le manifeste d'une réforme, sinon d'une révolution.

Ce n'est pas, avons-nous dit plus haut, un livre d'histoire des sciences ou de mathématiques de telle ou telle spécialisation. Non, à coup sûr. Mais c'est un livre bourré d'informations historiques et mathématiques très sûres, et qui bouscule toutes les spécialisations. Partout il faut arrondir, composer des pourcentages, évaluer des probabilités, et l'aventure des approximations numériques est pleine de paradoxes et de pièges, depuis très longtemps. Le savoir est indispensable. Quand on referme le livre on est convaincu qu'il y a, en effet, nécessité de réécrire et l'histoire des mathématiques et l'enseignement de celles-ci. On sait surtout que l'on tient en main un bréviaire auquel il faut revenir maintes fois, et qu'il est faux que la culture soit ce qui reste lorsqu'on a tout oublié.

Pierre Costabel.

" Jeux de réseaux », Cahiers S.T.S., 9-10, 1986.

Ce cahier est issu d'un séminaire sur les modèles utilisant la notion de réseaux, en informatique, physique, biologie, voire en sciences sociales. Il est très riche, et la plupart du temps très accessible. F. Fogelman fait le point sur les réseaux d'automates (automates cellulaires du jeu de la vie, automates booléens, automates à seuils). Atlan rappelle qu'un réseau booléen permet la « reconnaissance » d'une séquence pseudo-aléatoire. G. Weisbuch montre qu'un tel réseau peut, en modélisant l'interaction entre gènes, expliquer la génération d'équilibres ponctués, c'est-à-dire l'alternance de phases stables où les mutations sont inefficientes et de transitions vers un nouvel équilibre des populations. C. Michel traite des différentes approches du parallélisme dans la construction d'ordinateurs, le 
problème étant maintenant de pouvoir mixer les architectures à unique instruction sur multiples données (mais qui exigent des données mises en ordre) et celles à multiples instructions et multiples données. Il aurait pu se dispenser des analogies sociales entre totalitarisme et SIMD. M. Tchuente montre qu'un réseau centralisé a les meilleures performances de calcul, et un réseau décentralisé les meilleures performances de communication. E. Guyon et $\mathrm{C}$. Mitescu montrent comment les phénomènes de percolation dans un réseau peuvent recouvrir aussi bien la recherche d'un chemin dans un labyrinthe que la conductance, la diffusion d'un ou de plusieurs liquides en milieu poreux, voire la contagion bactérienne ou la croissance d'amas. J. de Rosnay compare les réseaux cristallins, neuronaux, immunitaires. I. Stengers rappelle que l'étude formelle des réseaux a montré que les réseaux écologiques pouvaient être stabilisés aussi bien à chaque niveau que globalement, selon les conditions, et $\mathbf{R}$. Chauvin que les insectes sociaux peuvent résoudre des problèmes par une coopération aléatoire, à condition d'être très nombreux. Y. Bouligand montre que les défauts des systèmes cristallins s'organisent eux-mêmes en super-réseaux. F. Varela que la modularité du système nerveux peut expliquer un comportement épileptoïde comme son organisation colonnaire. A. Kirman utilise les graphes stochastiques pour définir le seuil à partir duquel le noyau (non améliorable) d'une économie de coalition peut converger avec celui d'une économie de concurrence. Flament et Degenne rappellent que des intérêts antagonistes ne définissent pas strictement une partition de classe. A. Orléan met en parallèle la convention keynésienne, les anticipations rationnelles, et le mimétisme girardien. J.-P. Dupuy voit dans la panique de la foule et dans un marché où les équilibres sont pluraux, la même forme d'une communication entre éléments d'une totalité par l'intermédiaire du niveau de cette seule totalité. La notion de réseau se révèle partout être plus qu'une métaphore et induire des parentés de formalisme ou de problématique. Signalons seulement que la théorie des réseaux d'automates se développe très vite, et que la limitation à des interactions symétriques, encore indiquée ici, n'est déjà plus d'actualité. Bref un ensemble fort stimulant pour l'épistémologue.

Pierre LIVET.

Bernard D'ESPAGNAT, Une Incertaine réalité. Le monde quantique, la connaissance et la durée. Paris, Gauthier-Villars, 1985. 15,5 × 24, X + 310 p., bibliogr., index.

Les thèses de $B$. d'Espagnat sont maintenant bien connues et n'ont pas fondamentalement varié depuis $\dot{A}$ la recherche $d u$ réel. Avant de les rappeler succinctement, il faut marquer que, loin de tout artefact épistémologique, il pose à la totalité de la physique, mais en fonction du principe quantique et des difficultés introduites par les "Inégalités de Bell », une question spécifiquement philosophique : celle de la réalité à laquelle la science est censée accéder. Cette question est par définition " transcendantale » au sens large et authentique de 
ce mot, et n'implique pas nécessairement une réponse en termes de « réalisme transcendantal " que précisément l'auteur critique ici (p. 85 et suivantes). Les philosophes lui seront reconnaissants de les rappeler ainsi à une tâche plus importante (repenser le réel, la cause et le temps) que les descriptions et les conceptualisations épistémologiques. Sa réponse est nuancée et paraît parfois découler davantage d'un simple enregistrement des contradictions entre les physiques macroscopique, relativiste et quantique, que de la promotion d'une pıoblématique réellement nouvelle. L'enjeu est le réalisme de la physique l'Auteur le maintient et on devra le créditer sur ce point d'une probité « phénoménologique " et d'une fidélité aux réquisits ultimes de la science. Mais le réalisme classique et relativiste est attaqué, par la vérification expérimentale des Inégalités de Bell, sur son fondement même qui est la " localité » des phénomènes et des événements dans l'espace-temps. De là, devant l'impossibilité malgré tout d'abandonner la réalité « en soi » et de virer au phénoménisme, la nécessité de distinguer, au sein d'un « réalisme non-local », entre une réalité indépendante de l'homme et la réduction de celle-ci aux phénomènes exigés par la science et impliqués dans ses opérations techno-théoriques. L'Auteur fait l'inventaire des tentatives impossibles pour concilier le réalisme unitaire et les données de la microphysique. Il note quelques développements plus récents (théories de l'irréversibilité et de la complexité) qui suggèrent l'impossibilité d'échapper à l'ordre des phénomènes.

Les philosophes apprécieront la confiance que l'Auteur leur fait très régulièrement, ainsi que ses efforts pour libérer le réel ou l'Être des schèmes opératoires des objets et des équations, et retrouver ainsi, d'une manière quasi kantienne, un sens et un lieu pour la liberté, la causalité, la conscience, l'art, etc. Mais ils se demanderont comment il est possible de scinder ainsi en deux le réel et de le rendre " incertain "; de quelle différence ou scission il s'agit ici, et qui l'opère : la science elle-même devenue sujet transcendantal ? Un sujet tantôt physicien tantôt philosophe ex machina, etc. ? Il reste à dégager la " philosophie spontanée » de l'auteur.

François LARUELLE.

Joëlle Proust, Questions de forme : logique et proposition analytique de Kant à Carnap. Paris, Fayard, 1986. $14 \times 22,504$ p., bibliogr., index.

Une étude sur le concept d'analyticité ne pouvait être aujourd'hui que bienvenue et beaucoup seront tentés d'y chercher une initiation à la philosophie analytique. Pourtant, sans s'interdire d'éclairer son lecteur sur la genèse de cette école, l'auteur s'est fixé un autre but : examiner " le rôle que la notion de forme logique, et plus précisément du concept de proposition analytique, joue dans l'examen des conditions d'un savoir objectif » (p. XXIV). L'analytique, parent pauvre de la distinction introduite par Kant, ne faisant problème ni pour Frege ni jusqu'à un certain point pour Carnap, la matière pourrait sembler mince. En réalité, bien avant 1930 , une nouvelle idée de l'analyticité était à l'œuvre, dans le logicisme par exemple, et l'ouvrage en retrace la lente gestation. Le choix de 
La Syntaxe logique du langage comme terminus ad quem s'imposait donc et attirera peut-être l'attention sur un des textes majeurs de la philosophie contemporaine.

La première section cerne le lieu et les conditions d'apparition du couple analytique-synthétique et montre, en particulier, la place occupée dans la genèse de la philosophie transcendantale par un examen critique du projet leibnizien de caractéristique. Contre Leibniz qui ne concevait la marche de la pensée que comme une analyse, c'est-à-dire une résolution des concepts, Kant fait valoir que la composition n'est pas le simple inverse de la décomposition. La découverte, au-delà de l'usage externe, d'un usage interne du caractère interdit de concevoir le jugement comme un simple acte de comparaison. L'unité analytique du concept repose sur l'unité synthétique de l'aperception.

Des quatre auteurs étudiés dans l'ouvrage, Bolzano est le seul à avoir vu dans le concept de proposition analytique un problème et le lecteur sera reconnaissant à Madame Proust d'avoir réservé une section à ce philosophe sans postérité mais non sans originalité. Avec lui, l'analyticité, qui n'implique plus la vérité, dépend de trois conditions : la détermination préalable de la valeur de vérité, la variation et l'objectivité. Vraie ou fausse, une proposition sera analytique si ses éléments constitutifs peuvent varier sans que sa valeur de vérité soit modifiée, ni son objectivité détruite. La révision atteint aussi le concept de forme logique, qui n'est plus définie syntactiquement. La différence entre expressions catégorématiques et syncatégorématiques, partant entre la forme et la matière d'une proposition, est abolie et, dans une démarche qui n'est pas sans rappeler celle de Tarski, Bolzano construit un concept sémantique de validité, plus faible que celui de vérité. Mais, bien qu'il conclue lui aussi à la stérilité de l'analytique, sa théorie des vérités en soi reste précritique et le terme " onto-transcendantal " est introduit pour désigner cette "persistance de la recherche d'une condition générale a priori transposée dans un univers du discours qui admet des formes objectives "en soi" " (p. XVIII).

Les deux dernières sections retracent l'histoire de l'assaut mené contre le synthétique a priori. Prenant pour acquis la définition de l'analyticité, Frege cherche avant tout à dériver les mathématiques de la logique. Mais si les théorèmes de l'arithmétique sont analytiques, ce ne peut être au sens kantien et il faut donc remettre tacitement en chantier l'analyticité. L'essentiel porte sur le statut de la définition. S'il est vrai que celle-ci est une convention destinée à abréger le discours, elle ne saurait pour autant être arbitraire et c'est pourquoi le principe suprême des définitions stipulera que « les noms correctement construits doivent toujours dénoter quelque chose " (p. 206). Pour que l'enchaînement analytique soit fécond, deux conditions sont encore requises : distinguer le sens et la dénotation, et renoncer du même coup à la théorie de l'identité exposée en 1879 . Ainsi, alors que Frege croit parfaire la doctrine de Kant, il prive la philosophie transcendantale de son arrière-plan critique, puisque la reconstruction de l'arithmétique vise non plus à donner une connaissance certaine de cette science mais à en mettre en évidence l'essence.

Carnap a rebuté plus d'un lecteur car il fait dépendre'la solution des problèmes philosophiques de considérations dont il est très facile de perdre de vue la 
raison d'être. La règle que s'est fixée Madame Proust d'allier l'attention au détail et le souci de l'ensemble était plus que jamais nécessaire et ceux qui voudraient mieux comprendre comment l'analytique a pu en venir à délimiter le champ d'intervention de la philosophie trouveront une aide précieuse dans les quatre chapitres consacrés successivement à La Reconstruction logique du monde (1928) puis à La Syntaxe logique du langage (1934). Certes, la vérité logique apparaît désormais comme conventionnelle et l'analyticité comme relative à un langage. Mais les éventuels abus du principe de tolérance sont corrigés par la syntaxe générale qui garantit en particulier la traduction et la synonymie.

L'ouvrage se recommande par l'intérêt des questions qu'il aborde et le sérieux avec lequel il les traite. Ceux qui s'interrogent sur les rapports de la logique et de la philosophie liront avec profit les pages consacrées à la nature de la forme logique ou au statut de la définition et même ceux qui connaissent déjà Kant, Frege ou Carnap trouveront à s'y instruire. Mais si l'étude des auteurs est minutieuse et fidèle, l'orientation donnée à l'ensemble est plus discutable. L'unité de l'ouvrage est assurée par une double intention, dont l'une est comme le négatif de l'autre : contester le caractère empiriste de l'usage fait par Carnap de l'analyticité, montrer chez des philosophes considérés à un degré ou à un autre comme étrangers à la tradition kantienne la permanence de la question transcendantale. Dans le premier cas, l'auteur note à juste titre que le ralliement du Cercle de Vienne à l'empirisme n'était pas dépourvu d'intention polémique. Dans l'intérêt même de la raison, il eût été préférable, semble-t-il, de ne pas suivre l'adversaire sur ce terrain car il est à craindre que cette thèse provocatrice n'entretienne les vieux préjugés qui identifient l'empirisme au crime contre l'esprit, mais lui donnent aussi l'attrait du fruit défendu. Le concept de transcendantal présente l'avantage de ne pas prêter à ces équivoques. Il y a déjà longtemps que Reichenbach, dans sa préface à La Philosophie de l'espace et du temps, avait fait remarquer qu'à notre époque, le véritable héritage kantien était peut-être à chercher du côté de ceux qui maintenaient le dialogue avec la communauté scientifique. Il n'en reste pas moins que, comme son nom l'indique, la philosophie analytique pourrait très grossièrement être caractérisée par la volonté de bannir de son vocabulaire le substantif " synthèse " et plus encore son qualificatif « transcendantal ». On est en droit de penser que cet ostracisme a assez duré et ceux qu'asphyxie l'atmosphère raréfiée de la philosophie analytique accueilleront peut-être le retour du transcendantal comme une bouffée d'air frais. Mais les indications fournies sur ce transcendantal «transsystématique " que l'on propose de distinguer « de sa réalisation dans la philosophie critique de Kant » (p. 402) ne permettent pas de s'en former une idée claire. Le projet s'expose, en effet, à deux dangers : compromettre la spécificité du transcendantal, ou présenter la philosophie kantienne comme seule autorisée à poser les problèmes d'une philosophie de la connaissance. Malgré les corrections qu'apporte le contexte, il reste difficile d'admettre par exemple que « Bolzano et Frege fixent la question transcendantale pour lui fournir une autre réponse que celle qu'y apportait Kant " (p. XVIII ; cf. p. 262-263) ou que, de Kant au Carnap de l'Aufbau, « au fond, les questions considérées comme pertinentes et les types 
de solutions acceptables n'ont pas changé " (p. 327). Ces réserves n'affectent en rien les autres qualités d'un ouvrage dont tout donne à penser qu'il trouvera de nombreux lecteurs et leur donnera le goût de fréquenter les philosophes dont il $\mathrm{y}$ est question.

Michel BOURDEAU.

\section{HISTOIRE DES IDÉES}

D'Érasme à Campanella. Textes de Roland CrahaY, éd. par Jacques MARX. Bruxelles, Éd. de l'Université de Bruxelles, 1985. $16 \times 24,161$ p. (« Problèmes d'histoire du christianisme »).

Pour le grand érudit et l'esprit fin qu'est Roland Crahay, il n'y avait sans doute pas de meilleur hommage à lui rendre, dût sa modestie en souffrir, que de publier ces six textes de lui, si caractéristiques de ses méthodes de travail et de son champ principal de recherches, à l'occasion de sa retraite.

Ces six articles, prélevés sur sa riche bibliographie (une cinquantaine de titres, présentés au début du volume) ont été publiés originellement dans différents ouvrages ou revues. Érasme est depuis toujours l'un de ses " héros ». Mais ce qui le passionne en tant qu'historien des religions, et plus particulièrement de la censure dans l'Europe du Nord, c'est l'humaniste chrétien interdit de publication, tantôt par les censeurs de Louvain et les divers Index des Pays-Bas (Anvers, 1570 et 1571), succédant à l'Index tridentin de Pie IV en 1564, ou publié sous des cieux plus cléments (comme cette édition des Colloques de Dublin, en 1712, largement utilisée par la pédagogie protestante), ou contesté dans son évangélisme même (car il ne convient ni au formalisme dogmatique des théologiens et des prélats traditionnels, ni à l'esprit réformateur ou révolutionnaire de Luther et de son aile gauche). Esprit minutieux, R. Crahay ne fait jamais usage d'une formule - comme humanisme chrétien, ou évangélisme - sans l'analyser, en examiner les occurrences, en soupeser les synonymes ou les analogues. Il s'appuie sur des faits, développe des idées avec rigueur, fuyant les mots vagues ou approximatifs qui dispensent de penser droit.

Campanella, dont on attend avec impatience l'édition critique de La Cité $d u$ soleil, le passionne également pour ce mélange de rationalisme, d'utopisme et de générosité dans la construction de sa cité idéale. Esprit fort différent, mais également théoricien politique, Jean Bodin n'a cessé de retenir son intérêt : d'où cette analyse de sa République, qui interfère avec ses préoccupations relatives aux censures religieuses. Enfin, une autre utopie a plus d'une fois sollicité $R$. Crahay : celle des anabaptistes, ces mal-aimés, ces persécutés des différentes Églises établies, ces révolutionnaires assoiffés de 